\title{
ANÁLISIS TÉRMICO DE GEOSINTÉTICOS UTILIZADOS EN LA REHABILITACIÓN DE PAVIMENTOS
}

\section{THERMAL ANALYSIS OF GEOSYNTHETICS USED IN THE REHABILITATION OF PAVEMENTS}

\author{
José Norambuena-Contreras $^{1} \quad$ David Zamora Barraza $^{2} \quad$ Daniel Castro-Fresno $^{3}$ \\ Ángel Vega-Zamanillo ${ }^{4}$
}

Recibido 3 de marzo de 2008, aceptado 8 de enero de 2009

Received: March 3, 2008 Accepted: January 8, 2009

\begin{abstract}
RESUMEN
El crecimiento de la utilización de los geosintéticos en la rehabilitación de pavimentos flexibles se ha visto incrementado de forma considerable desde la década de los años setenta. Estos materiales presentan una serie de ventajas que motivan su gran demanda en la actualidad. La utilización de geosintéticos en la rehabilitación se basa en su capacidad de resistir deformaciones, atenuando las fisuras y grietas que pudiesen presentar los pavimentos flexibles. Se han realizado dos experiencias en laboratorio para simular el proceso de puesta en obra de los geosintéticos en la zona de intercapa. El procedimiento consiste en verter sobre el geosintético extendido, una capa de mezcla bituminosa en caliente comprendida entre $135-165^{\circ} \mathrm{C}$. El análisis térmico de los resultados obtenidos avala significativamente al material poliéster (PS), con respecto al polipropileno (PP), en futuras aplicaciones de rehabilitación de pavimentos flexibles.
\end{abstract}

Palabras clave: Mezcla bituminosa, geosintéticos, polímero, termoplástico.

\begin{abstract}
The use of geosynthetics in rehabilitating flexible pavements has substantially expanded since the mid-seventies. These materials have a number of advantages that motivate their demand at present. The use of geosynthetics in rehabilitation is based on its ability to resist deformation, attenuating fissures and cracks that flexible pavements could present. Two experiments have been done to simulate the process of placing the geosynthetics in the interlayer area. The process consists of pouring on the spread geosynthetics a layer of hot bituminous mix between 135-165 ${ }^{\circ} \mathrm{C}$. The thermal analysis of the results shows a significant performance of polyester (PS), compared to polypropylene (PP), in future applications for the rehabilitation of flexible pavements.
\end{abstract}

Keywords: Asphalt mixture, geosynthetics, polymer, thermoplastic.

\section{INTRODUCCIÓN}

El concepto genérico de geosintético designa un producto en el que al menos uno de sus componentes está fabricado a base de polímeros sintéticos o naturales. Los geosintéticos se fabrican en forma de fieltro, manto, lámina o estructura tridimensional, los cuales son puestos en contacto con el suelo u otros materiales en aplicaciones de la ingeniería civil [1]. La industria de geosintéticos incorpora un alto grado de polímeros como principal materia de fabricación, para así asegurar una correcta deformación y resistencia ante las solicitaciones a las cuales se enfrentará su producto. Se pueden clasificar en productos fabricados a partir de procedimientos de extrusión (geoplásticos), productos que

\footnotetext{
1 Grupo de Investigación de Tecnología de la Construcción. Departamento de Transportes y Tecnología de Proyectos y Procesos. Universidad de Cantabria. Avda. de los Castros s/n. Santander. España. E-mail: jernesto.norambuena@unican.es

2 Grupo de Investigación de Tecnología de la Construcción. Departamento de Transportes y Tecnología de Proyectos y Procesos. Universidad de Cantabria. Avda. de los Castros s/n. Santander. España. E-mail: dzamora@ucm.cl

3 Grupo de Investigación de Tecnología de la Construcción. Departamento de Transportes y Tecnología de Proyectos y Procesos. Universidad de Cantabria. Avda. de los Castros s/n. Santander. España. E-mail: daniel.castro@unican.es

4 Grupo de Caminos de Santander. Departamento de Transportes y Tecnología de Proyectos y Procesos. Universidad de Cantabria. Avda. de los Castros s/n, Santander. España. E-mail: angel.vega@unican.es
} 
incluyen en su fabricación tecnología textil (geotextiles) y productos formados por ambas tecnologías: textil y plástica, como los geocompuestos [2]. Por otra parte, se pueden encontrar los tejidos o no tejidos, que se componen normalmente de termoplásticos como el polipropileno o poliéster, cuyas denominaciones químicas internacionales corresponden a PP y PS.

El proceso de puesta en obra de estos productos en la zona de intercapa consiste en verter sobre el geosintético extendido una capa de mezcla bituminosa en caliente a una temperatura comprendida en un rango de $135-165^{\circ} \mathrm{C}$; [3-4], como se muestra en la figura 1.

Según su composición, los geosintéticos como cuerpos poliméricos son en mayor o menor medida susceptibles a los cambios de temperatura [5], pudiendo variar sus propiedades en función de la exposición a la misma; de ahí que estudiar su comportamiento térmico sea fundamental desde el punto de vista del uso en rehabilitación de firmes, que impulsa su contacto con mezclas bituminosas en caliente a altas temperaturas.

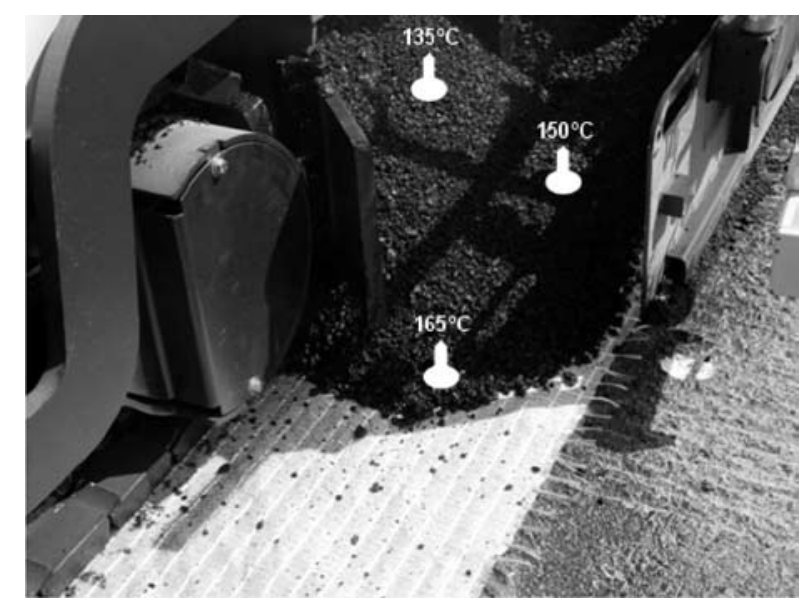

Figura 1. Vertido de mezcla bituminosa en caliente.

Por ello, este estudio plantea el análisis del comportamiento térmico de los geosintéticos utilizados en la rehabilitación de pavimentos flexibles, valorando las prestaciones del material polimérico constituyente.

\section{Objetivos}

1. Simular en el laboratorio del Grupo de Caminos de Santander (GCS), el proceso de puesta en obra de los geosintéticos en la intercapa.

2. Analizar el material polimérico constituyente de los geosintéticos, frente a sus temperaturas de fusión y transición vítrea.

\section{ANÁLISIS TEÓRICO}

\section{Metodología}

La clasificación de los materiales seleccionados para las experiencias de laboratorio correspondió a geosintéticos de poliéster y polipropileno, clasificados según se muestra en la tabla 1.

La metodología de ensayo utilizada fue de dos tipos:

Experiencia 1): Medición de la evolución de la temperatura del geosintético, adquirida por convección al estar en contacto con aire caliente a $150^{\circ} \mathrm{C}$. Esta medición permite obtener la temperatura máxima de referencia adquirida en función del tiempo de exposición al aire.

Experiencia 2): Medición de la variación de la superficie del geosintético, debida a la adquisición de temperatura por conducción al estar en contacto con un material granular caliente (a $135{ }^{\circ} \mathrm{C}$ y $165^{\circ} \mathrm{C}$ ). Esta experiencia permite determinar la variación media del área, en función del tiempo de exposición con el material granular caliente.

Tabla 1. Geosintéticos seleccionados para los ensayos y su clasificación en tipos.

\begin{tabular}{|l|c|}
\hline \multicolumn{1}{|c|}{$\begin{array}{c}\text { GEOSINTÉTICOS } \\
\text { SELECIONADOS }\end{array}$} & $\begin{array}{c}\text { CLASIFICACIÓN } \\
\text { DE LAS PROBETAS }\end{array}$ \\
\hline Geomalla de poliéster [PS] & TIPO A \\
\hline Geomalla de poliéster [PS] & TIPO B \\
\hline $\begin{array}{l}\text { Geotextil geocompuesto no tejido } \\
\text { de polipropileno reforzado con } \\
\text { fibra de vidrio [PP] }\end{array}$ & TIPO C \\
\hline $\begin{array}{l}\text { Geotextilno tejido de polipropileno } \\
\text { [PP] }\end{array}$ & TIPO D \\
\hline $\begin{array}{l}\text { Geotextil no tejido punzonado } \\
\text { 100\% polipropileno [PP] }\end{array}$ & TIPO E \\
\hline
\end{tabular}

Todas las probetas fueron cortadas con una sección cuadrada de $100 \mathrm{~cm}^{2}$. Las condiciones de clima del laboratorio para las experiencias correspondió a una temperatura ambiente de $22{ }^{\circ} \mathrm{C}$ y una humedad relativa fluctuante entre un $45 \%$ y $50 \%$. Las probetas fueron acondicionadas a temperatura ambiente 24 horas antes de cada experiencia.

Para la experiencia 1), las probetas fueron del Tipo A y Tipo C (tabla 1), seleccionadas como representación de los materiales poliéster [PS] y polipropileno [PP], respectivamente. Una vez cortadas fueron atadas a un pasador por medio de un sistema de ganchos metálicos, permitiendo así una sujeción más eficaz que evitase desprendimientos. 
Preparadas las probetas de ensayo, estas fueron introducidas en un horno, el cual se encontraba a una temperatura de $150{ }^{\circ} \mathrm{C}$, temperatura media del intervalo al cual se puede encontrar la mezcla en caliente una vez es puesta en obra. Las temperaturas adquiridas por cada probeta fueron medidas en intervalos continuados de 5 minutos en un período de 1 hora de exposición (figura 2).

Una vez conocida la temperatura de referencia máxima adquirida por convección, valores máximos obtenidos en la experiencia 1), se procede a simular mediante la experiencia 2) el comportamiento que tendría cada uno de los cinco tipos de geosintéticos seleccionados (tabla 1) al estar en contacto con la mezcla bituminosa en caliente.

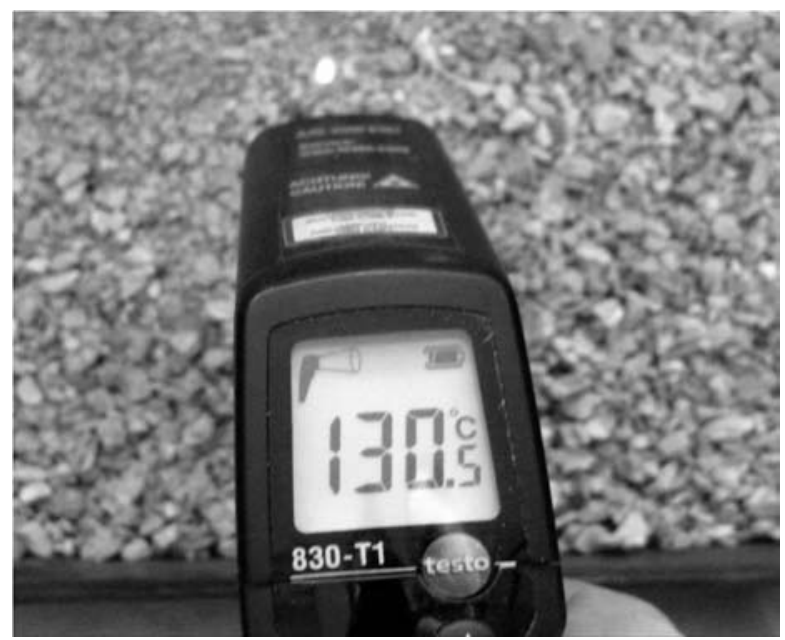

Figura 2. Toma de datos de experiencia 1).

Así, para simular el proceso de puesta en obra de la mezcla bituminosa en caliente sobre el geosintético, permitiendo así evaluar los cambios sufridos por él, se empleó de un material granular caliente y un recubrimiento de papel aluminio.

Para la experiencia 2) se prepararon 12 probetas de cada uno de los 5 geosintéticos a ensayar, repitiendo el proceso en 2 ocasiones para cada una de las 2 temperaturas evaluadas, $135{ }^{\circ} \mathrm{C}$ y $165{ }^{\circ} \mathrm{C}$. Resultando, por tanto, un total de 240 probetas ensayadas.

Las probetas se cubrieron con papel de aluminio convencional (figura 3), previamente a su colocación sobre las camas de arena de 2,5 cm de espesor extendidas en bandejas metálicas de laboratorio.

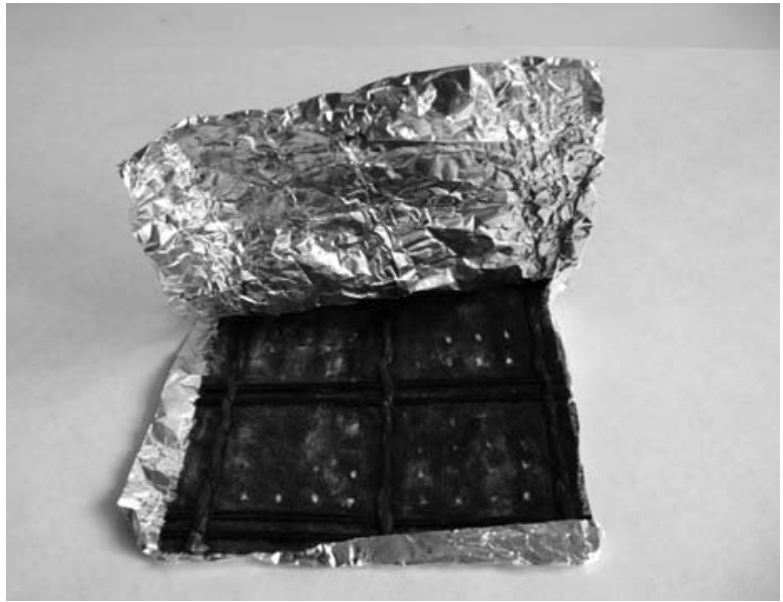

Figura 3. Preparación de probetas.

Las bandejas y el material granular se encontraban acondicionadas en un horno a la correspondiente temperatura de ensayo con 24 horas de antelación (Figura 4). Posteriormente las probetas eran recubiertas por una segunda capa de arena a la misma temperatura que la primera, simulando así el vertido de la mezcla en caliente en su etapa de instalación. El papel de aluminio sirvió para preservar la integridad de la muestra, aumentando además la transmisión de calor del material granular al geosintético, tal y como lo haría en la realidad el betún en caliente retenido $[6,7]$.

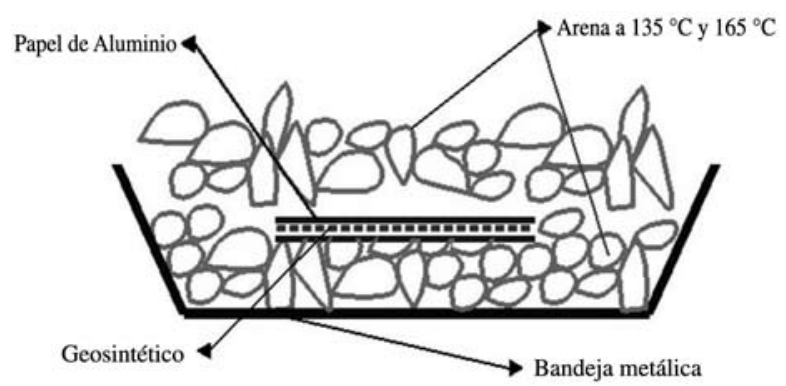

Figura 4. Esquema de la experiencia 2).

La toma de datos de las variaciones de área producidas en las probetas fue medida en 12 intervalos, cada 5 minutos durante un período de 1 hora, retirando una probeta de cada tipo y comparando sus dimensiones con la correspondiente probeta testigo sin ensayar.

Los instrumentos y equipos utilizados para el desarrollo de los experimentos y la obtención de datos fueron: bandejas metálicas, tenazas, termómetro infrarrojo marca TESTO ${ }^{\circledR}$, modelo 830-T1 con una precisión de $0,1{ }^{\circ} \mathrm{C}$ y exactitud de $\pm 5^{\circ} \mathrm{C}$, y horno de laboratorio de aire forzado marca SELECTA $^{\circledR}$ modelo $374 \mathrm{~A}$ con una temperatura máxima de generación de $180{ }^{\circ} \mathrm{C}$. 


\section{RESULTADOS Y ANÁLISIS}

La figura 5 muestra los resultados obtenidos en la experiencia 1). Como era de esperar, se observa un aumento progresivo de la temperatura con el tiempo de exposición al aire caliente. La muestra Tipo $\mathrm{C}$ adquiere el máximo de temperatura de $128{ }^{\circ} \mathrm{C}$ en un intervalo de tiempo menor, en 15 minutos, al correspondiente a la temperatura máxima alcanzada en la muestra Tipo A, que es de $126{ }^{\circ} \mathrm{C}$. Por tanto el valor de temperatura máxima de referencia es $128^{\circ} \mathrm{C}$, el cual supone un $85 \%$ de la temperatura del aire $\left(150^{\circ} \mathrm{C}\right)$. Esto significa una pérdida mínima de un $15 \%$ en la transmisión de temperatura al geosintético por convección.

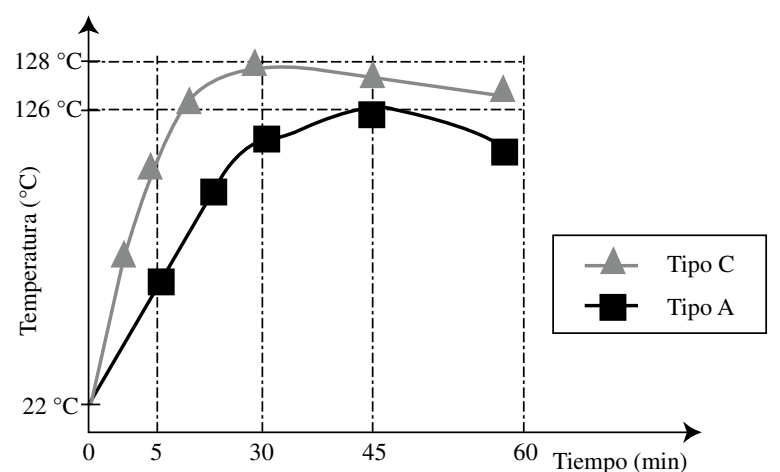

Figura 5. Temperatura adquirida por convección en horno a $150{ }^{\circ} \mathrm{C}$.

Por otra parte, en la figura 6 y figura 7 se muestran los valores medios de las variaciones porcentuales de la superficie con respecto a sus áreas originales, correspondientes a la experiencia 2), a las temperaturas de 135 y $165{ }^{\circ} \mathrm{C}$ respectivamente. El valor máximo correspondió a un $15 \%$ para la temperatura de $135{ }^{\circ} \mathrm{C}$ (Probetas C), como de un $86 \%$ para la temperatura de $165{ }^{\circ} \mathrm{C}$ (Probetas D) mostrada en figura 10.

Por otra parte, los mínimos registrados fueron de un $8 \%$ para $135^{\circ} \mathrm{C}$ (Probetas A) y de un $22 \%$ para una temperatura de $165^{\circ} \mathrm{C}$ (Probetas A), según se muestra en figura 11, indicando una variación porcentual considerable de los geosintéticos ensayados por conducción.

Este comportamiento registrado en las variaciones porcentuales del área de los geosintéticos, puede ser analizado desde el punto de vista del material polimérico constituyente, como posible absorbente de deformaciones apreciables frente a fenómenos térmicos.

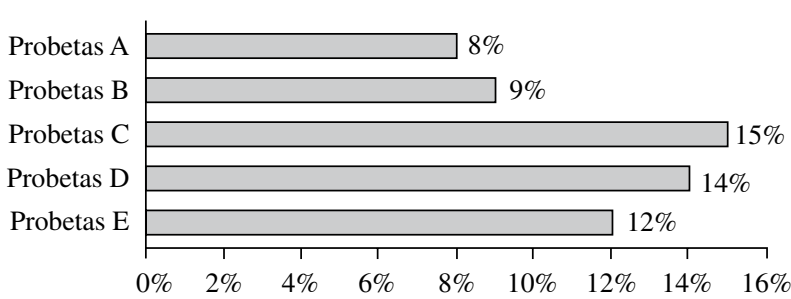

Figura 6. Variación porcentual media del área de las probetas adquirida por conducción a $135^{\circ} \mathrm{C}$.

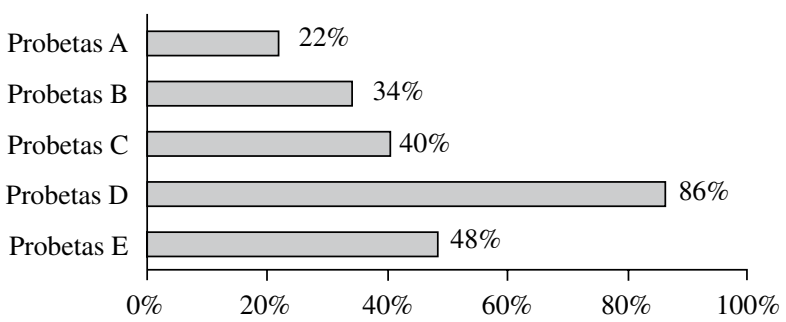

Figura 7. Variación porcentual media del área de las probetas adquirida por conducción a $165^{\circ} \mathrm{C}$.

Los polímeros pueden asumir diferentes formas geométricas de relativa complejidad de acuerdo a su estructura de ligaduras cruzadas, cortas o bien mediante cadenas ramificadas de variada orientación molecular [8] (figura 8), llamadas también áreas semicristalinas, prevaleciendo constante un rango aleatorio entre macromoléculas, conocidas como zonas amorfas (figura 9).

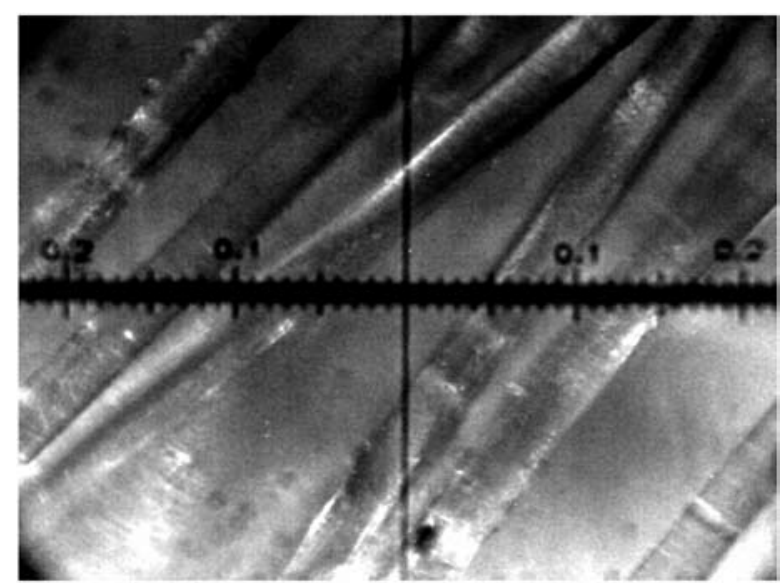

Figura 8. Microfotografía de fibras de geotextil no tejido de polipropileno [PP] ensayado a $135^{\circ} \mathrm{C}, 100$ $\mu \mathrm{m}$.

Los geosintéticos estudiados corresponden al grupo de polímeros lineales que se conocen con el nombre de termoplásticos, tales como el polipropileno PP (figura 10) y el poliéster PS (figura 11). Estos geosintéticos reaccionan frente a los cambios de temperatura, "seudotransición termodinámica $\mathrm{Tg}$ " provocando contracciones en su estructura interna [9]. 


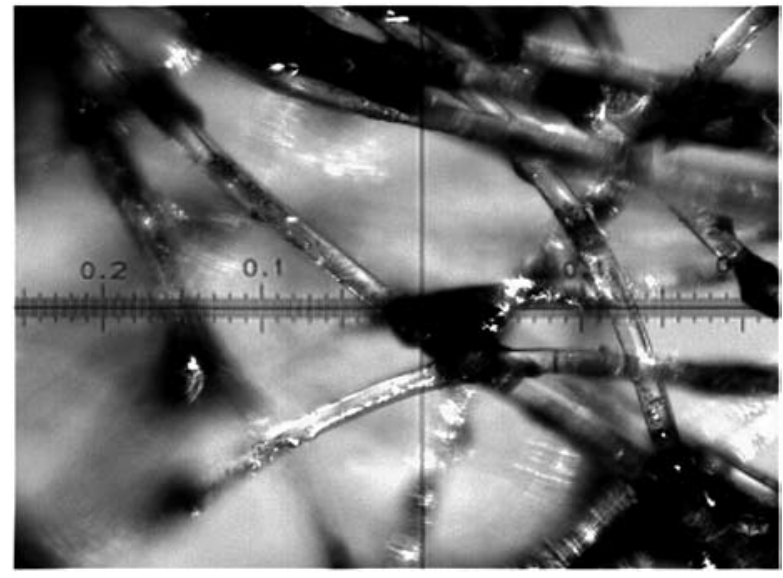

Figura 9. Microfotografía de fibras de geomalla de poliéster [PS] ensayada a $135^{\circ} \mathrm{C}, 100 \mu \mathrm{m}$

La temperatura de transición vítrea (Tg) mostrada en la figura 12 e indicada anteriormente, señala el cambio del material de un estado frágil a otro dúctil; es decir, deja de ser rígido comenzando a ser ahulado o blando.

Con valores de temperatura superiores a la transición vítrea ( $\mathrm{Tg}$ ) los enlaces secundarios de las moléculas son mucho más débiles que el movimiento térmico de las mismas, por ello el polímero se torna ahulado, adquiriendo cierta elasticidad y capacidad de deformación plástica sin fractura. Cabe destacar que este comportamiento es específico de polímeros del tipo termoplásticos [9].

Por otra parte, la temperatura de fusión (Tm) corresponde a la situación en donde las cadenas se mueven libremente, iniciando el cambio de fase a un estado líquido [10]. Una variación del gradiente térmico de mayor a menor valor genera en las cadenas un empaquetamiento $\mathrm{y}$, en consecuencia, un elevado grado de ordenación, causando la estabilidad interna del material. Este fenómeno se presentaba en el instante que las probetas eran retiradas de la intercapa de las camas de arena, siendo expuestas a temperatura ambiente (figura 10).

En la figura 12 se observa el comportamiento termogravimétrico teórico de las Probetas A y B (poliéster, PS), ensayadas en los intervalos de $\mathrm{Tg}\left(70{ }^{\circ} \mathrm{C}\right)$ y $\mathrm{Tm}$ $\left(240-260{ }^{\circ} \mathrm{C}\right.$ ) a las temperaturas de $135^{\circ} \mathrm{C}$ y $165^{\circ} \mathrm{C}$, así también las de polipropileno PP.

Se observa que un material termoplástico como el PS sometido a las temperaturas de ensayo descritas no supera la pérdida total ni parcial de las propiedades del material ensayado. Esto es debido a que el rango de temperaturas (Tm) de las probetas se encontraba lejos de ser superado por las temperaturas de ensayo, permitiendo que las moléculas del material polimérico constituyente no alcanzaran a experimentar movimientos excesivos. Este comportamiento aseguró que no se generase un estado líquido, sino un reordenamiento interno o empaquetamiento de contracción, el cual en consecuencia proporcionó una escasa movilidad y disminución de su área, comportándose así como un material en excelentes condiciones termogravimétricas.

Por otra parte el comportamiento teórico de las Probetas $\mathrm{C}$, D y E (polipropileno, PP), ensayadas en los intervalos de $\mathrm{Tg}\left(-10^{\circ} \mathrm{C}\right)$ y $\mathrm{Tm}\left(140-160^{\circ} \mathrm{C}\right)$ a $135^{\circ} \mathrm{C}$ y $165^{\circ} \mathrm{C}$ exceden el rango de temperaturas (Tm), causando así la movilidad libre de moléculas en suspensión, generándose de esta manera un estado líquido, el cual iba disminuyendo según el tiempo y la temperatura de exposición. En un material termoplástico de estas características, el cambio durante la transición es gradual, conllevando a la pérdida total o parcial de las propiedades, causando así un reblandecimiento apreciable ligado a su comportamiento termogravimétrico (figura 12).

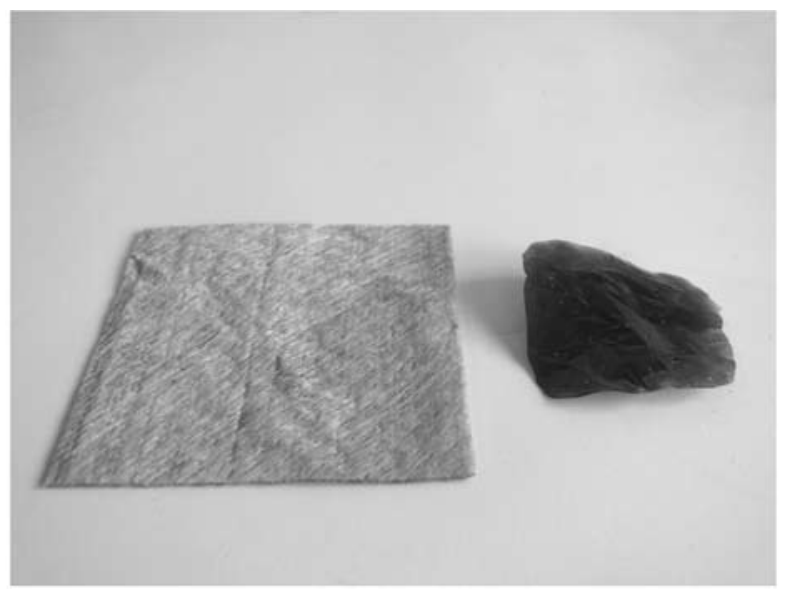

Figura 10. Probeta $\mathrm{D}$, geotextil de polipropileno ensayado a $165^{\circ} \mathrm{C}$.

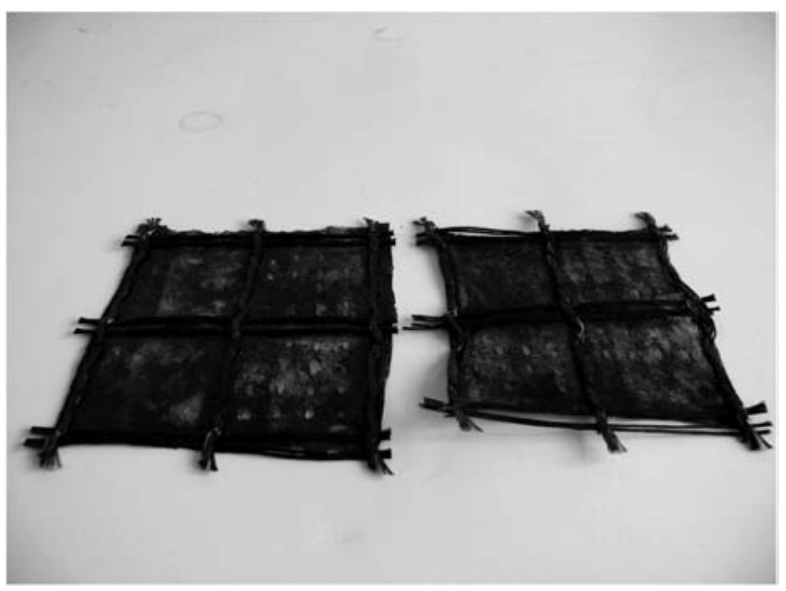

Figura 11. Probeta A, geomalla de poliéster ensayada a $165^{\circ} \mathrm{C}$. 


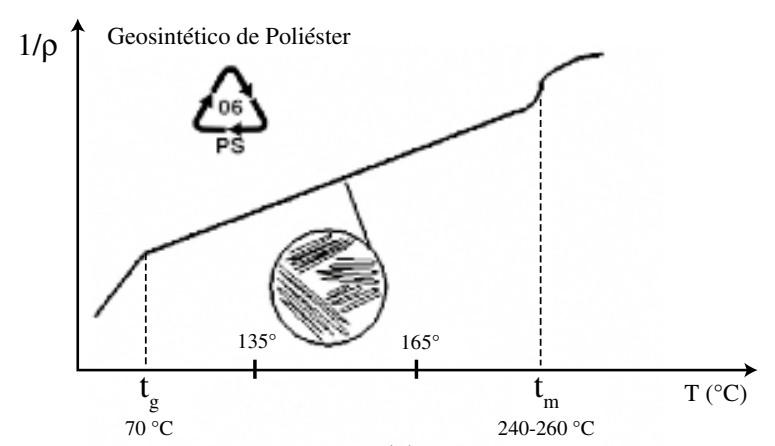

(a)

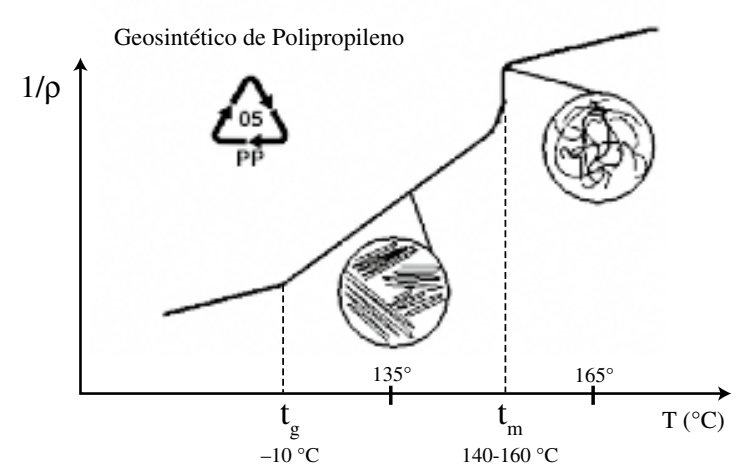

(b)

Figura 12. Comportamiento termogravimétrico del (a) poliéster y (b) polipropileno en exposición a temperaturas de ensayo.

\section{CONCLUSIONES}

Los geosintéticos que se componen estructuralmente por un alto contenido polimérico de polipropileno PP, tienden a adquirir con mayor facilidad el calor que se les transmite. Por otra parte, los geosintéticos de poliéster PS tardan más en realizar la misma captación de calor trasmitida por convección.

La variación media del área de las probetas ensayadas a temperaturas de 135 y $165{ }^{\circ} \mathrm{C}$ permite determinar el comportamiento térmico de un geosintético previo conocimiento de la temperatura vítrea y de fusión del constituyente polimérico principal del material, parámetros claves en el control de sus temperaturas una vez son instalados en obra.

El análisis térmico y el comportamiento termogravimétrico experimentado por los materiales ensayados avalan significativamente al material poliéster PS, con respecto al polipropileno PP, en futuras aplicaciones de rehabilitación de pavimentos flexibles, cuyo recapado corresponda a mezcla bituminosa en caliente.
En resumen, no se aconseja usar geosintéticos de polipropileno PP con mezclas bituminosas en caliente por encima de los $140{ }^{\circ} \mathrm{C}$. No obstante, sí puede usarse en geosintéticos de poliéster PS, hasta temperaturas de $165^{\circ} \mathrm{C}$.

\section{REFERENCIAS}

[1] R. Holtz, B.R. Christopher and R.R. Berg. "Geosynthetic desing and construction guidelines". Rep. No. FHWA Hi-95-038, National Highway Institute, Federal Highway Administration, US Department of Transportation, USA. 1998.

[2] R.M. Koerner. "Durability and Aging of Geosynthetics”. Elsevier Applied Science. 1989.

[3] J.W. Button and R.L. Lytton. "Guidelines for using geosynthetics with hot-mix asphalt overlays to reduce reflective cracking". Transp Res Rec. 111-119. 2007.

[4] J.W. Button and R.L. Lytton. "Guidelinas for using geosynthetics with HMA overlays to reduce reflective cracking". Rep. $\mathrm{N}^{\circ}$ 1777-P2, Information $\&$ Technology exchange center (ITEC), Texas. 2003.

[5] S. Vanden Eynde, G.W.H. Mathot, Höhne, J.W. K, Schawe and H. Reynaers. "Thermal behaviour of homogeneous ethylene-1-octene copolymers and linear polyethylene at high pressures". Polymer. Vol. $41 \mathrm{~N}^{\circ}$ 9, pp. 3411-3423. 2000.

[6] A. De Bondt. "Introduction. Anti-reflective Cracking Desing of Reinforced (Reiforced) Asphaltic Overlays". Thesis, Delft University of Technology. Holanda. 1999.

[7] D. Castro and F. Ballester. "Influence of asphalt cement type and oven type of asphalt retention capacity of paving geotextiles". Geosynth. Int., Vol. $13 \mathrm{~N}^{\circ}$ 2, pp. 83-86. 2006.

[8] Y.G. Hsuan. "Approach to the study of durability of reinforcement fibers and yarns in geosynthetic clay liners". Geotext.Geomembr. Vol. $20 \mathrm{~N}^{\mathrm{o}} 1$, pp. 63-76. 2002.

[9] V.B. Mathot. "Calorimetry and Thermal Analysis of Polymers". Hanser Publishers, Munich Vienna New York. 1994. 